INVITED ARTICLE

\title{
Kerosene, Camphor, and Naphthalene Poisoning in Children
}

\author{
Surjeet Kumar ${ }^{1}$, TK Kavitha ${ }^{2}$, Suresh K Angurana ${ }^{3}$
}

\begin{abstract}
Kerosene poisoning is one of the most common accidental poisoning in children in developing countries due common use of kerosene in household and unsafe storage practices. Aspiration pneumonitis is the most common manifestation of kerosene ingestion due to its low viscosity, high volatility, and low surface tension. The treatment of aspiration pneumonitis due to kerosene poisoning is symptomatic including oxygen support, respiratory monitoring, and careful monitoring of fluid balance. Children with severe respiratory distress and hypoxemia unresponsive to supplemental oxygen and/or severe central nervous system involvement require early intubation and mechanical ventilation. Transfer to the pediatric intensive care unit (PICU) is required at this stage. Emesis, gastric lavage, and administration of activated charcoal are contraindicated due to risk of aspiration. There is no clear benefit of using corticosteroids or prophylactic antibiotics. Asymptomatic children should be kept under observation for atleast 6 hours after exposure. The mortality rate is low and death occurs due to pneumonitis. Camphor is used in household items including vaporized or topical cold preparations, liniments, moth repellents, for performing rituals in religious ceremonies, and in antimicrobial preparations. Camphor poisoning is not very common in childhood. Even small doses of camphor can cause serious toxicity and is potentially fatal. The onset of action is very rapid (5-15 minutes). The common manifestations are confusion, restlessness, delirium, and hallucinations, muscle twitching, myoclonus, ataxia, hyperreflexia, fasciculations, and seizures. Seizures are common and serious complication in camphor toxicity. The treatment is supportive including decontamination, gastric lavage, activated charcoal, and seizure control. Naphthalene is a major constituent of mothballs which are commonly used in household to protect clothes from moths. Though the poisoning with naphthalene is uncommon in children, most of the cases with naphthalene poisoning occur in developing countries where mothballs are still commonly used. The manifestations of naphthalene toxicity are predominantly due to acute intravascular hemolysis leading to anemia, hemoglobinuria, methemoglobinemia, and acute kidney injury (AKI). The treatment of naphthalene toxicity is supportive in form of transfusion of the packed red blood cells, monitoring of fluid and electrolyte balance, administration of alkalis in presence of hemoglobinuria, and renal replacement therapy. Prevention is better than cure. The strategies should be adopted to prevent children being exposed to these toxic compounds in the house-hold. Safe storage of toxic compounds away from the reach of children, avoiding storing kerosene in cold drink and beverage bottles, community education, provision of electricity in rural areas, safe cooking practices, and parental supervision are important interventions to prevent accidental poisoning among children.
\end{abstract}

Keywords: Aspiration pneumonitis, Hemoglobinuria, Hemolysis, Seizures.

Indian Journal of Critical Care Medicine (2019): 10.5005/jp-journals-10071-23316

\section{Kerosene Poisoning}

\section{Introduction}

Kerosene is a straight-chain or aliphatic hydrocarbon commonly used in households and industries, especially in rural areas in developing countries like ours. It is used in households for cooking, lighting, heating, and in paints and pesticides. Exposure occurs accidently in children when they consume the kerosene stored in juice bottles, beverages bottles, and colorful packing within their reach. ${ }^{1,2}$ The risk factors for kerosene oil poisoning in children are unsafe storage, inadequate supervision, inadequate house space, low socioeconomic status, lack of maternal education, and lack of family support. ${ }^{1,3}$ The developed countries have largely eliminated the use of kerosene and hence accidental kerosene ingestions; unfortunately, it is still common in developing countries like India especially in rural areas. ${ }^{4-6}$ Kerosene poisoning is one of the commonest accidental poisonings in children accounting for $14-60 \%$ of admissions due to accidental poisoning in children..$^{3,5-10}$ Most common complication is aspiration pneumonitis. The treatment of aspiration pneumonitis is symptomatic in the form of oxygen support, respiratory monitoring, and lung-protective mechanical ventilation in case of worsening lung condition. The mortality due to kerosene ingestion is low $(<1 \%)$, but it is associated with high morbidity due to respiratory involvement. ${ }^{1-3}$ Kerosene oil poisoning in children is a preventable cause of significant mortality and morbidity by safe storage of kerosene in house out of reach

\begin{abstract}
${ }^{1-3}$ Division of Pediatric Critical Care, Department of Pediatrics, Advanced Pediatrics Centre, Postgraduate Institute of Medical Education and Research (PGIMER), Chandigarh, India

Corresponding Author: Suresh K Angurana, Division of Pediatric Critical Care, Department of Pediatrics, Advanced Pediatrics Centre, Postgraduate Institute of Medical Education and Research (PGIMER), Chandigarh, India, Phone: +91 9855373969, e-mail: sureshangurana@ gmail.com
\end{abstract}

How to cite this article: Kumar S, Kavitha TK, Angurana SK. Kerosene, Camphor, and Naphthalene Poisoning in Children. Indian J Crit Care Med 2019;23(Suppl 4):S278-S281.

Source of support: Nil

Conflict of interest: None

of children, community education, provision of electricity in rural areas, and safe cooking practices by using cooking gas instead of kerosene stoves. ${ }^{1,2}$

\section{Pathophysiology}

Kerosene oil is having very low viscosity, high volatility, and low surface tension. The toxicity of kerosene also depends on its constituents naphthenic and aromatic hydrocarbons. Aspiration pneumonitis is the most common manifestation of kerosene toxicity. ${ }^{1,2}$ Due to its low viscosity, low surface tension, and high 
volatility, there are high chances of aspiration, which usually occurs at the time of ingestion (coughing and gagging) or vomiting (spontaneous or induced) after ingestion. Aspiration of even small amounts of kerosene $(<1 \mathrm{~mL})$ can lead to serious, potentially lifethreatening toxicity. Kerosene once aspirated spread rapidly across the surfaces of airway and alveoli leading to inactivation of type II pneumocytes, and resulting surfactant deficiency, intra-alveolar hemorrhage, inflammation, and necrosis. ${ }^{1,11}$ All these can lead to surfactant dysfunction, poor lung compliance, and acute respiratory distress syndrome. ${ }^{11}$

Chemical pneumonitis does not result from dermal absorption or ingestion in the absence of aspiration. Kerosene is poorly absorbed through the gastrointestinal tract (GIT) but often causes irritation of the gastrointestinal mucosa. Sometimes, there may be symptoms due to central nervous system involvement. ${ }^{11}$

\section{Clinical Manifestations}

Most of the poisoning with kerosene is unintentional. Males are more commonly involved, and the majority of the cases are $<5$ years of age. ${ }^{1,4,7}$ Commonest complication after kerosene ingestion or inhalation is chemical pneumonitis. A history of coughing or gagging during ingestion is consistent with aspiration. Coughing, rapid breathing, retractions, increased work of breathing, wheezing are common symptoms of aspiration pneumonitis. Bronchospasm may contribute to ventilation-perfusion mismatch and exacerbate hypoxia. ${ }^{3,6,11}$ There may be cyanosis. Respiratory symptoms develop within minutes of ingestion (usually within 1 hour). It almost always begins and evolves within the first 6 hours of ingestion. Hemorrhagic pulmonary edema and respiratory arrest occurs within 24 hours, and worsening continues till 24-48 hours, followed by progressive improvement. If symptoms do not develop within 6 hours of ingestion, the patients will be normal. Respiratory involvement ranges from mild symptoms or rapid progression to acute respiratory distress syndrome (ARDS) and respiratory failure. ${ }^{2,6,11}$

After significant oral ingestion, there may be gastrointestinal symptoms, including nausea, vomiting, diarrhea, and abdominal pain. ${ }^{1,3}$ Vomiting increases the risk of aspiration. Acute kerosene exposure by inhalation or large ingestion can lead to central nervous system symptoms resulting in headache, dizziness, drowsiness, euphoria, restlessness, ataxia, poor ventilatory drive, convulsions, coma, and occasionally death. Central nervous system (CNS) symptoms can also occur due to hypoxemia and acidosis due to respiratory involvement. ${ }^{1}$ Fever is seen in up to $30 \%$ cases, and it does not correlate with clinical symptoms and does not always imply bacterial superinfection. Defervescence occurs within 24 hours in 3/4th cases. Persistence of fever $>48-72$ hours should point toward bacterial infection. ${ }^{4,11}$

\section{Laboratory Investigations}

A chest radiograph is indicated in all children with a history of kerosene ingestion and respiratory symptoms. Chest radiographs may be normal initially but may show abnormalities within 6 hours of aspiration in the majority of symptomatic cases. Chest radiographic abnormalities may occur as soon as 20 minutes or as late as 24 hours after ingestion. Typical radiographic findings are increased bronchovascular markings and infiltrate involving bibasilar and perihilar regions. Lobar consolidation, pneumothorax, pneumomediastinum, and pleural effusion are uncommon. Chest radiographs can remain abnormal long after clinical improvement. Pneumatoceles can develop 2-3 weeks after exposure., ${ }^{6,11,12}$
Arterial blood gas analysis is important to know about oxygenation and ventilation status in children with respiratory distress as well as the need for escalation of respiratory support and response to it. Based on the severity of the illness, the acid-base status, electrolyte balance, complete blood count, and liver and renal function test need to be followed.

\section{Treatment}

The mainstay of treatment for kerosene poisoning is supportive care. Early diagnosis and treatment of aspiration pneumonitis reduce mortality. ${ }^{2}$ Remove the patient from exposure and remove soiled clothing. Wash the contaminated skin or eyes with soap and water. In the case of accidental ingestion, the emesis and gastric lavage are contraindicated as there is a higher risk of aspiration than systemic absorption. Activated charcoal is not beneficial because it does not bind kerosene and may induce vomiting. ${ }^{11}$

There is no clear role of corticosteroids or prophylactic antibiotics. ${ }^{11-13}$ Antibiotics are reserved for children with definite evidence of infection. Fluid administration should be restricted to maintenance requirements to minimize the risk of pulmonary edema. Children with bronchospasm should be treated with nebulized beta2 agonists. ${ }^{11}$

If aspiration pneumonitis develops, the respiratory treatment is supportive. The airway patency should be evaluated and established. Any child with respiratory symptoms (grunting, tachypnea, or cyanosis) should be started on humidified oxygen and requires an arterial blood gas analysis. Oxygen support, respiratory monitoring, intake and output monitoring, and supportive care are important management strategies. It is important to realize that children with respiratory compromise at presentation to the emergency department can suffer rapid deterioration. ${ }^{4,5,11}$

For children who have severe distress with hypoxemia unresponsive to supplemental oxygen and/or severe central nervous system involvement require early intubation and mechanical ventilation. ${ }^{3,11}$ Cuffed endotracheal tube is preferred to prevent aspiration. Conventional mechanical ventilation with lung-protective ventilation strategies including low tidal volume $(6 \mathrm{~mL} / \mathrm{kg})$, use of adequate positive end expiratory pressure (PEEP), limiting plateau pressure to $<30 \mathrm{~mm} \mathrm{Hg}$, inspiratory: expiratory ratio of $1: 2$ to $1: 1$, oxygen target of $\mathrm{SpO}_{2} 88-95 \%$ and $\mathrm{PaO}_{2} 55-80 \mathrm{~mm}$ $\mathrm{Hg}$, and permissive hypercapnia should be used to limit ventilatorinduced lung injury. In the case of no improvement, high-frequency ventilation and extracorporeal membrane oxygenation help in the management of respiratory failure and ARDS associated with severe pneumonitis. ${ }^{11}$

\section{Disposition}

Children who have ingested small amounts of kerosene and developed no symptoms of aspiration (choking, coughing, vomiting, and respiratory distress), normal oxygen saturation, or other features can be discharged after an observation period of 6 hours after ingestion and advised to attend hospital if symptoms develop. Child should be hospitalized if symptomatic at presentation, or develops symptoms within the 6 hour period of observation. If respiratory compromise or hypoxemia is present, admission to the pediatric intensive care unit is indicated. ${ }^{11}$

\section{Prevention}

Kerosene poisoning in children is a preventable cause of significant mortality and morbidity. Safe storage of kerosene in household out of reach of children, avoiding storing kerosene in cold drink 
and beverage bottles, community education, and provision of electricity in rural areas, and safe cooking practices by using cooking gas instead of kerosene stoves. The word poison should be written prominently on kerosene containing containers. ${ }^{1,2,5,8,13}$

\section{Camphor Poisoning}

\section{Introduction}

Camphor is a colorless substance with a strong odor and pungent taste, available in solid and liquid forms. It is commonly seen in household items, including vaporized or topical cold preparations (for example Vicks Vaporub is $4.18 \%$ camphor and Tiger Balm), topical musculoskeletal anesthetics preparations (liniments), moth repellents, for performing rituals in religious ceremonies, and in antimicrobial preparations. ${ }^{14}$ Camphor is also used in the Indian household in the form of small cubes for its fragrance.

Though camphor poisoning is not very common in childhood, ${ }^{10}$ the easy availability in various forms put children at high risk of camphor poisoning. Even small doses of camphor can cause severe toxicity and are potentially fatal. ${ }^{15}$ Camphor content should not exceed $11 \%$ in camphor containing products [as per United States Food and Drug Administration (FDA)], but the concentration of camphor is not mentioned on the majority of the products. ${ }^{14,16}$ The onset of action is very rapid, and the main effect occurs on the central nervous system, and seizures are most common. The treatment if supportive including, decontamination, gastric lavage, activated charcoal, and seizure control. ${ }^{13}$

\section{Pathophysiology}

Camphor is a rapidly acting neurotoxin and can lead to excitation followed by depression of CNS. It is rapidly absorbed from GIT, leading to the rapid onset of action of toxic effects within 5-20 minutes, and peak effect occurs at 90 minutes. Thus, the onset of neurotoxicity is very rapid after ingestion. ${ }^{17}$ Major systemic toxicity has not been reported with ingestion of up to $30 \mathrm{mg} / \mathrm{kg}$ of camphor. The neurotoxic dose of camphor is $>50 \mathrm{mg} / \mathrm{kg}$ body weight, and the fatal dose is $500 \mathrm{mg} / \mathrm{kg}$ body weight. ${ }^{14,17,18}$

\section{Clinical Features}

Clinical symptoms begin and progress rapidly (within 5-15 minutes of ingestion) in form of a feeling of generalized warmth which progresses to burning sensation in pharynx and epigastrium and vomiting followed by changes in mental status (confusion, restlessness, delirium, and hallucinations), muscle twitching, myoclonus, ataxia, hyperreflexia, fasciculations, and seizures. There may be a strong smell from the breath. Seizures are the most common reported symptom after camphor ingestion. Seizure may be the first sign of systemic toxicity and may occur soon after ingestion without any preceding symptoms. ${ }^{14,17,19}$ Seizures can occur after gastrointestinal, dermal, or inhalation exposures to camphor. ${ }^{16}$ Camphor can also lead to hepatic and renal injury. Mortality usually occurs due to seizures or respiratory failure. ${ }^{14}$

\section{Treatment}

Management of camphor ingestion consists of supportive care with a focus on airway management and seizure control. Skin and ocular decontamination should be done by flushing with large amount of water. Cases with camphor inhalation should be moved to fresh air. It is better to avoid the induction of emesis. The use of ipecac is not preferred because of the potential for seizures. As camphor is rapidly absorbed from the stomach, gastric lavage and activated charcoal have a limited role in the management of camphor ingestion. In camphor poisoning, seizures occur mostly soon after ingestion (maximum within the first 2 hours). Seizures should be managed with benzodiazepines (midazolam and lorazepam), and repeat doses may be needed. For uncontrolled seizures, a second anticonvulsant is needed, such as phenobarbitone or phenytoin. For refractory status epilepticus, continuous infusions of midazolam and propofol are preferred. Hemodialysis is of not much benefit. ${ }^{14,17,19}$

\section{Disposition}

Children with signs and symptoms of camphor toxicity and those who have ingested $>30 \mathrm{mg} / \mathrm{kg}$ body weight should be admitted to the hospital, preferably to pediatric intensive care unit (PICU). In the event of seizure, children require PICU admission and observation for a minimum of 2 days. ${ }^{17}$ Asymptomatic cases need to be observed for a minimum period of 6 hours and can be discharged if he/she remains asymptomatic after 6 hours. ${ }^{17}$

\section{Naphthalene Poisoning}

\section{Introduction}

Naphthalene is a major ingredient in mothballs, which are commonly used as a household pesticide to protect clothes from moths. It sublimates, and the fume has insecticidal properties. The naphthalene content of one mothball varies from 0.5 to $5 \mathrm{~g}^{20}$ The fatal dose of naphthalene in children is unknown, but even one mothball can result in toxicity, and there are reports of deaths following the ingestion of naphthalene balls. ${ }^{20}$ Naphthalene has been replaced with 1,4-dichlorobenzene (which is harmless) in most countries, and mothballs have been banned. But in our country, mothballs are still commonly used, they are poorly labeled, and users are often not aware of their contents. Most of the poisoning with mothballs occur in developing countries. ${ }^{20,21}$

The absorption of naphthalene from GIT is erratic in the pediatric population. Other routes of exposure are inhalational and skin contact. Naphthalene is rapidly absorbed when inhaled. Dermal absorption may occur in infants, and it may be further enhanced by prior application of oils. ${ }^{20}$ Naphthalene poisoning is uncommon in children due to its pungent smell, taste, water insolubility, and poor absorption from GIT. $^{20}$ The small size of mothballs and the presence of color may attract the attention of children and lead to accidental ingestion. ${ }^{4}$ The manifestations of naphthalene toxicity are predominantly due to acute intravascular hemolysis leading to anemia, hemoglobinuria, and acute kidney injury (AKI). ${ }^{22}$ The treatment of supporting including transfusion of packed red blood cells, monitoring of fluid and electrolyte balance, administration of alkalis in the presence of hemoglobinuria, and renal replacement therapy. ${ }^{20}$

\section{Pathophysiology}

Naphthalene is an oxidant and ingestion lead to acute intravascular hemolysis particularly in children with glucose-6-phosphate dehydrogenase (G6PD) deficiency as they have a low tolerance to oxidative stress. ${ }^{21,22}$ The red blood cells have various mechanisms to prevent toxicity from oxidative stress, and most common being the generation of reduced nicotinamide adenine dinucleotide phosphate (NADPH) from hexose monophosphate shunt. The NADPH is helpful to replenish adequate stores of reduced glutathione, which prevent hemolysis from oxidative stress. Children with G6PD deficiency do not produce adequate NADPH 
and have low levels of glutathione and are at higher risk of hemolysis, methemoglobinemia, and acute kidney injury after exposure to naphthalene. ${ }^{20}$

Oxidative and mechanical trauma to RBCs leads to the release of free hemoglobin into plasma and which is filtered in the glomerulus and is incorporated into proximal convoluted tubules where intracellular hemoglobin dissociates into heme and globin. The increased levels of intracellular heme are cytotoxic and lead to AKI by decreasing renal perfusion, direct cytotoxicity, and intratubular casts formation (interaction of heme proteins with Tamm-Horsfall protein). Heme is highly lipophilic, oxidant, proinflammatory, and apoptotic, which contributes to its cytotoxic effects, and mitochondria are particularly vulnerable to heme mediated cytotoxicity. ${ }^{20}$

\section{Clinical Features}

The clinical manifestations of naphthalene poisoning include headache, vomiting, diarrhea, abdominal pain, fever, and altered mental status. ${ }^{20}$ The most significant clinical manifestations in naphthalene poisoning occur due to hemolysis leading to anemia, jaundice, hemoglobinuria, methemoglobinemia, and acute kidney injury. Hemolysis can be acute or slowly progressive and even delayed. ${ }^{20,22}$

\section{Laboratory Investigations}

Complete blood count, peripheral blood film for evidence of hemolysis, urine and plasma hemoglobin, lactate dehydrogenase (LDH), renal and liver function tests, acid-base status, urine routine examination, methemoglobin levels, and G6PD levels need to be monitored in children with naphthalene poisoning.

\section{Treatment}

There is no specific treatment for naphthalene poisoning. Administration of milk, oils, and fats should be avoided as they may increase the absorption of naphthalene. The management is symptomatic, including transfusion of packed red blood cells for anemia, monitoring of fluid and electrolyte balance, administration of alkalis in presence of hemoglobinuria to prevent its deposition in renal tubules, and renal replacement therapy. ${ }^{20,22}$

\section{Prevention}

Store mothballs and boxes of mothballs out of reach of children. Wash clothing and bedding that has been stored in mothballs before wearing or using them. Use mothballs containing paradichlorobenzene instead of naphthalene because it is less harmful.

\section{References}

1. Dayasiri M, Jayamanne SF, Jayasinghe CY. Kerosene oil poisoning among children in rural Sri Lanka. Int J Pediatr 2017;2017:8798610. DOI: 10.1155/2017/8798610.

2. Parekh U, Gupta S. Kerosene-a toddler's sin: A five years study at tertiary care hospital in western India. J Forensic Leg Med 2017;47: 24-28. DOI: 10.1016/j.jflm.2017.02.004.

3. Venkatesh C, Sriram P, Adhisivam B, Mahadevan S. Clinical profile of children with kerosene aspiration. Trop Doct 2011;41(3):179-180. DOI: 10.1258/td.2011.110093.
4. Tshiamo W. Paraffin (kerosene)* poisoning in under-five children: a problem of developing countries. Int J Nurs Pract 2009;15(3):140-144. DOI: 10.1111/j.1440-172X.2009.01748.x.

5. Jayashree M, Singhi S. Changing trends and predictors of outcome in patients with acute poisoning admitted to the intensive care. J Trop Pediatr 2011;57(5):340-346. DOI: 10.1093/tropej/fmq099.

6. Basu M, Kundu TK, Dasgupta MK, Das DK, Saha I. Poisoning, stings and bites in children-- what is new? An experience from a tertiary care hospital in Kolkata. Indian J Public Health 2009;53(4): 229-231.

7. Roy MP, Gupta R, Bhatt M, Aggarwal KC. Profile of children hospitalized with acute poisoning in New Delhi. Indian Pediatr 2017;54(3):246-247. DOI: 10.1007/s13312-017-1039-9.

8. Dayasiri M, Jayamanne SF, Jayasinghe CY. Patterns and outcome of acute poisoning among children in rural Sri Lanka. BMC Pediatr. 2018;18(1):274. DOI: 10.1186/s12887-018-1246-0.

9. Kohli U, Kuttiat VS, Lodha R, Kabra SK. Profile of childhood poisoning at a tertiary care centre in North India. Indian J Pediatr 2008;75(8): 791-794. DOI: 10.1007/s12098-008-0105-7.

10. Peshin SS, Gupta YK. Poisoning due to household products: A ten years retrospective analysis of telephone calls to the National Poisons Information Centre, All India Institute of Medical Sciences, New Delhi, India. J Forensic Leg Med 2018;58:205-211. DOI: 10.1016/ j.jflm.2018.07.005.

11. Makrygianni EA, Palamidou F, Kaditis AG. Respiratory complications following hydrocarbon aspiration in children. Pediatr Pulmonol 2016;51(6):560-569. DOI: 10.1002/ppul.23392.

12. Das S, Behera SK, Xavier AS, Selvarajan S. Prophylactic Use of Steroids and Antibiotics in Acute Hydrocarbon Poisoning in Children. J Pharm Pract 2018; 897190018771520. DOI: 10.1177/0897190018771520.

13. Balme KH, Zar H, Swift DK, Mann MD. The efficacy of prophylactic antibiotics in the management of children with kerosene-associated pneumonitis: a double-blind randomised controlled trial. Clin Toxicol 2015;53(8):789-796. DOI: 10.3109/15563650.2015.1059943.

14. Sahana KS, Rajiv D. Camphor poisoning. Indian Pediatr 2012;49(10): 841-842. DOI: 10.1007/s13312-012-0174-6.

15. Matteucci MJ. One pill can kill: assessing the potential for fatal poisonings in children. Pediatr Ann 2005;34(12):964-968. DOI: 10.3928/0090-4481-20051201-12.

16. Khine H, Weiss D, Graber N, Hoffman RS, Esteban-Cruciani N, Avner JR. A cluster of children with seizures caused by camphor poisoning. Pediatrics 2009;123(5):1269-1272. DOI: 10.1542/peds.2008-2097.

17. Mathen PG, Sreekrishnan TP, Kumar KPG, Mohan N. Camphor Poisoning: A Rare Cause of Acute Symptomatic Seizures in Children. J Emerg Trauma Shock 2018;11(3):228-229. DOI: 10.4103/JETS. JETS_21_18.

18. Agarwal A, Malhotra HS. Camphor ingestion: an unusual cause of seizure. J Assoc Physicians India 2008;56:123-124.

19. Manoguerra AS, Erdman AR, Wax PM, Nelson LS, Caravati EM, Cobaugh DJ, et al. Camphor Poisoning: an evidence-based practice guideline for out-of-hospital management. Clin Toxicol 2006;44(4):357-370. DOI: 10.1080/15563650600671696.

20. Ekambaram S, Chandan Kumar KM, Mahalingam V. Acute kidney injury: A rare complication of mothball (Naphthalene) poisoning. Saudi J Kidney Dis Transpl 2017;28(6):1412-1415. DOI: 10.4103/13192442.220858.

21. Rahman MM, Mogni Mowla SG, Rahim A, Chowdhury FR, Jahan $S$, Hasan MN. Severe haemolytic anaemia due to ingestion of naphthalene (mothball) containing coconut oil. J Coll Physicians Surg Pak 2012;22(11):740-741. DOI: 11.2012/JCPSP.740741.

22. Uthuman AAA, Jayasinghe CS, Fernando AHN. Acute intravascular hemolysis due to naphthalene toxicity: a case report. J Med Case Rep 2019;13(1):91. DOI: 10.1186/s13256-018-1963-x. 